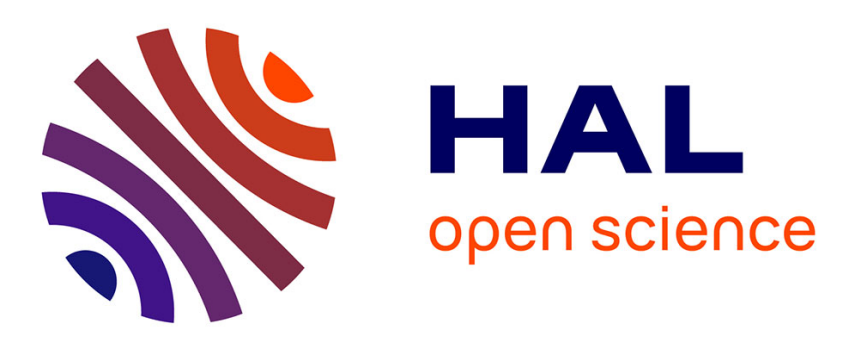

\title{
Time-Resolved Study of the Photoswitching of Gold Nanorods Coated with a Spin-Crossover Compound Shell
}

Ngoc Minh Tran, Marlène Palluel, Nathalie Daro, Guillaume Chastanet, Eric

Freysz

\section{To cite this version:}

Ngoc Minh Tran, Marlène Palluel, Nathalie Daro, Guillaume Chastanet, Eric Freysz. Time-Resolved Study of the Photoswitching of Gold Nanorods Coated with a Spin-Crossover Compound Shell. Journal of Physical Chemistry C, 2021, 125 (41), pp.22611-22621. 10.1021/acs.jpcc.1c03218 . hal03374297

\section{HAL Id: hal-03374297 \\ https://hal.science/hal-03374297}

Submitted on 12 Oct 2021

HAL is a multi-disciplinary open access archive for the deposit and dissemination of scientific research documents, whether they are published or not. The documents may come from teaching and research institutions in France or abroad, or from public or private research centers.
L'archive ouverte pluridisciplinaire HAL, est destinée au dépôt et à la diffusion de documents scientifiques de niveau recherche, publiés ou non, émanant des établissements d'enseignement et de recherche français ou étrangers, des laboratoires publics ou privés. 


\title{
Time-resolved study of the photoswitching of gold nanorods coated with spin crossover compound shell
}

\author{
Ngoc Minh Tran, ${ }^{\dagger}$ Marlène Palluel, ${ }^{\ddagger}$ Nathalie Daro, ${ }^{\ddagger}$ Guillaume Chastanet,,$\stackrel{\ddagger}{\ddagger}$ and \\ Eric Freysz $z^{* \dagger}$ \\ $\dagger$ †niv. Bordeaux, CNRS, LOMA, UMR 5798, F-33405 Talence, France \\ $\ddagger$ Univ. Bordeaux, CNRS, Bordeaux INP, ICMCB, UMR 5026, F-33600 Pessac, France \\ E-mail: eric.freysz@u-bordeaux.fr \\ Phone: +33 (0)540008313
}

\begin{abstract}
We investigate the spin-state photoswitching process in Au@SCO nanohybrids made of gold nanorods coated by a spin crossover (SCO) shell of polymeric triazole based $\mathrm{Fe}(\mathrm{II})$ complexes of $\left[\mathrm{Fe}(\mathrm{Htrz})_{2}\right.$ trz $]\left(\mathrm{BF}_{4}\right)$. Our research is performed exciting our samples with near-infrared quasi-continuous wave or nanosecond pulse, tunable from visible to near-infrared range. Time-resolved experiments ranging from millisecond down to nanosecond time-scales reveal the dynamics during the spin-state transition. Upon excitation, the thermal photoswitching of the sample happens within hundreds of milliseconds for quasi-continuous wave excitation. It happens within tens of nanoseconds for a nanosecond pulse. Compared to the SCO $\left[\mathrm{Fe}(\mathrm{Htrz})_{2} \operatorname{trz}\right]\left(\mathrm{BF}_{4}\right)$ nanoparticles powder, the peak power of quasi-continuous wave or fluence of the nanosecond laser required to induce the spin state transition in the nanohybrid is reduced. A
\end{abstract}


simple model that well accounts for the experimental results is proposed. We show that the experiments performed using nanosecond pulses make it possible to analyze the photo-switching within individual nanoparticles whereas the ones performed with quasi-continuous wave excitation reveal the photo-switching within an ensemble of nanoparticles. 


\section{Introduction}

Spin crossover $(\mathrm{SCO})$ is a phenomenon that occurs in families of transition metal complex in the center of which a metallic ion such as $\mathrm{Fe}(\mathrm{II}), \mathrm{Co}(\mathrm{II})$ is inserted. ${ }^{1-3}$ Upon variations of temperature, pressure or light intensity, the spin state of the metallic center may change, giving rise to a mechanism called spin crossover. ${ }^{4}$ It results in sizable modifications of the mechanical, magnetic, electrical and optical properties of the complexes. ${ }^{4-9}$ At the macroscopic scale, this change may be accompanied by a first-order phase transition which is often associated with a thermal hysteresis loop. Within the hysteresis loop and depending on its cycling history, the sample may remain in its high temperature (high spin - HS) or low temperature (low spin - LS) state as long as the temperature is kept within the thermal hysteresis loop. The potential application of this phenomenon in displays, indicators, data recording, and sensors, has triggered a lot of attention ${ }^{5,10-14}$ and numerous efforts have been made to design SCO compounds with well-suited properties. For that purpose, much attention has been driven towards polymeric triazole based $\mathrm{Fe}(\mathrm{II})$ complexes such as $\left[\mathrm{Fe}(\mathrm{Htrz})_{2} \mathrm{trz}\right]\left(\mathrm{BF}_{4}\right) .{ }^{15-17}$ The latter exhibits a broad thermal hysteresis loop centered above room temperature. Small nanostructures of these compounds can be synthesized while maintaining the cooperativity. ${ }^{18-23}$ Besides, it is possible to obtain plenty of different and exotic nanostructures based on such systems. ${ }^{24}$

However, for a lot of applications, an improvement in the response time of SCO compounds is required. Through conventional heating, it is usually difficult to record a spin state transition on a short time scale. To circumvent this problem attempts have been made using the light-induced excited spin-state trapping (LIESST) effect ${ }^{25}$ or local shockwave excitation. ${ }^{26}$ However, the LIESST effect is usually recorded at low temperatures ${ }^{27}$ and forbiddens applications at room temperature. Besides, the shock waves are usually induced through absorption of the laser pulse exciting the medium and therefore difficult to control and optimize. ${ }^{28}$ To go further, we have used the photo-thermal effect to rapidly switch a spin crossover compound between the LS and HS states. ${ }^{29}$ A fast photo-switching is recorded exciting the 
SCO compound with nanosecond laser pulses. ${ }^{30}$ The photo-switching time depends on the duration and energy of the optical pulse, as well as the thermal diffusion within and between the particles. ${ }^{31,32}$ This photo-switching is fastened in SCO nanoparticles (NPs). ${ }^{33}$ To further minimize the laser pulse energy required to photo-switch SCO NPs, a new approach based on a combination of gold and SCO NPs has been recently proposed. ${ }^{34-38}$ Along this line, we have recently demonstrated that insertion of gold nanorods (AuNRs) in [Fe(Htrz) $\left.)_{2} \operatorname{trz}\right]\left(\mathrm{BF}_{4}\right)$ NPs largely reduces the optical peak power required to induce the spin-state transition in these SCO nanohybrids. ${ }^{36,37}$ For simplicity we will hereby refer the NPs of $\left[\mathrm{Fe}(\mathrm{Htrz})_{2}\right.$ trz] $\left(\mathrm{BF}_{4}\right)$ and AuNRs embedded in $\left[\mathrm{Fe}(\mathrm{Htrz})_{2}\right.$ trz $]\left(\mathrm{BF}_{4}\right)$ as SCONP and $\mathrm{Au} @ \mathrm{SCO}$, respectively. Embedding AuNRs inside the SCONP provides a robust structure and good core/shell contact. ${ }^{37,38}$ The high and broad optical absorption of Au@SCO in the visible range results in burgundy color and permits to excite the $\mathrm{Au} @ \mathrm{SCO}$ in a large spectral range. Besides an optical excitation at $770 \mathrm{~nm}$, near the longitudinal SPR absorption peak of the AuNRs provides a means of selectively heating the core of the AuNRs. These Au@SCO are therefore expected to have optimized response time and low power consumption. ${ }^{37}$ In this paper we investigate the photoswitching process in these Au@SCO nanohybrids. The study is performed exciting the samples with near-infrared quasi-continuous wave (QCW) or nanosecond pulse tunable from visible to near-infrared range. These QCW and nanosecond pulse excitations permit studying the growing of the HS state within the samples under Heaviside's or Dirac's excitations, respectively. Upon Dirac excitation with nanosecond pulses a photo-switching time down to $20 \mathrm{~ns}$ is recorded in Au@SCO. We also show these nanosecond experiments give power to analyze the growing of the HS state fraction within an ensemble of individual SCONP and Au@SCO. Besides, we evidence that compared to the SCONP of the same size, the energy of the optical pulses required to induce the spin state transition in Au@SCO is overall reduced. A more remarkable reduction is recorded around the surface plasmon resonance (SPR) of the AuNRs. 


\section{Methods}

\section{General}

Transmission electron microscopy (TEM) images are acquired using a JEOL 1400+ (Japan), with a high-resolution mode and a tension of $60 \mathrm{kV}$. Each compound is analyzed separately and the measurements of 100 particles are done in order to give the average dimensions. Magnetic measurements are performed using a MPMS-5S Quantum Design SQUID magnetometer operating at $20 \mathrm{kOe}$. The powder samples are prepared in a thin layer ( $0.9 \mathrm{mg}$ on around $100 \mu \mathrm{m}$ thickness). The sample is weighted before any measurement and the diamagnetic contributions of the sample and the sample holder are removed. The thermal cycles are performed at around $0.7 \mathrm{Kmn}^{-1}$ scan rate. The absorption measurements are carried out shining the sample with a broadband halogen light (Thorlabs OSL1-EC) source and recording the spectra of the transmitted light with and without the sample with a compact spectrometer (Ocean Optics, Flame Vis-NIR) versus the temperature. The relative optical density is evaluated by computing the logarithmic ratio of the sample spectra over the reference spectra transmitted by a microscope glass slide.

\section{Millisecond photoswitching set-up}

The set-up used to record photoswitching kinetic of the samples at the millisecond time scale is displayed in Fig. 1a. The sample is kept in a thermally regulated oven and excited by a continuous wave (CW) laser diode (Thorlabs FPL785S) that central wavelength is about $785 \mathrm{~nm}$. The latter is almost within the longitudinal SPR absorption mode of Au@SCO. A mechanical shutter is used to generate optical pulses of steady state intensity with duration between $2 \mathrm{~ms}$ and $500 \mathrm{~ms}$. The exciting pulse is focused on the sample on slightly elliptical spot which diameter is about $0.8 \mathrm{~mm}$. The probe light is provided by a high-intensity halogen lamp (Thorlabs OSL1-EC) which spectrum ranges from $350 \mathrm{~nm}$ to $800 \mathrm{~nm}$. On the

sample, the halogen light is also focused on a spot of about $0.8 \mathrm{~mm}$ in diameter. The light 

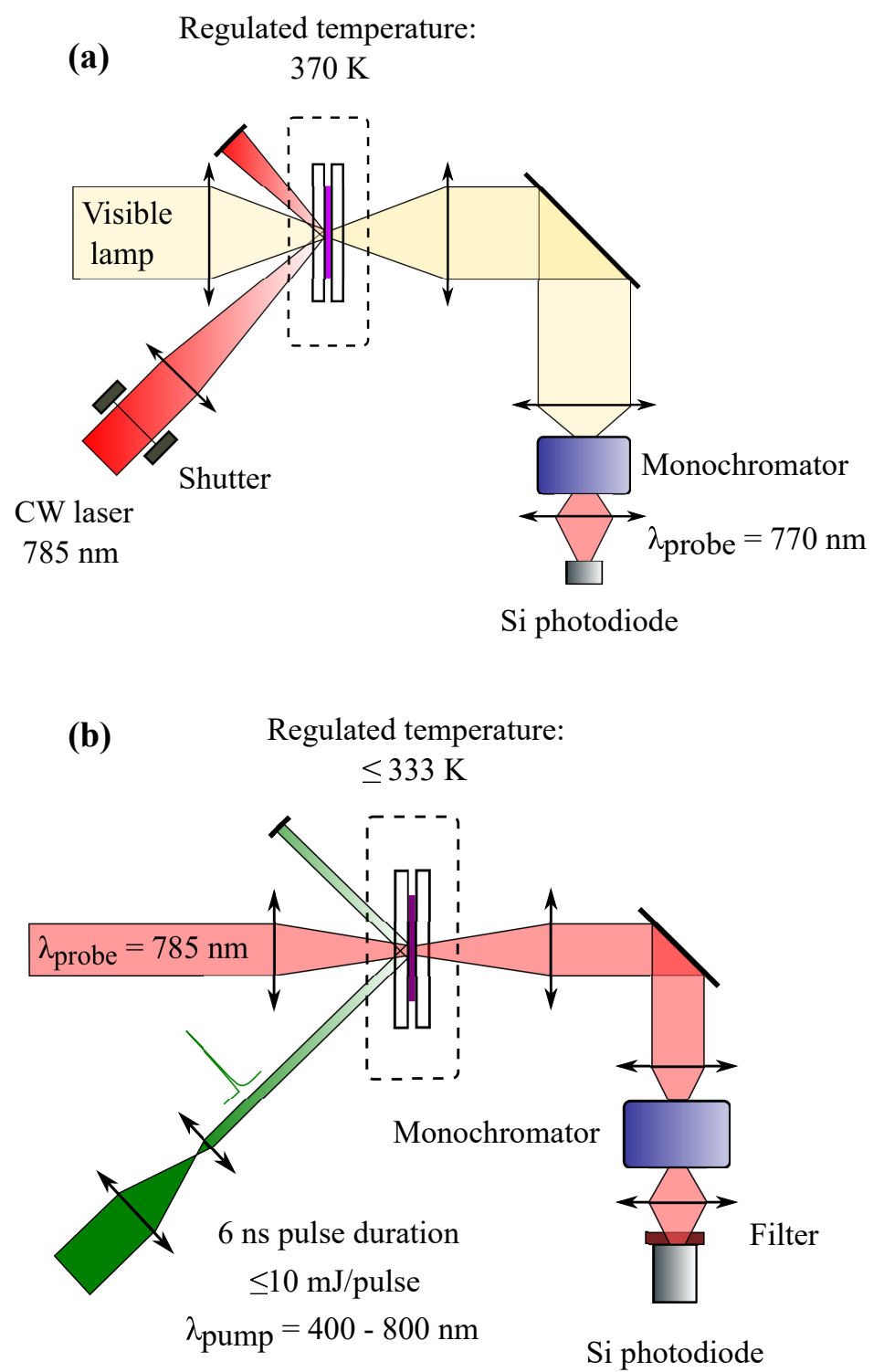

Figure 1: (a) Scheme of time-resolved transmission set-up using quasi continuous-wave light source. (b) Scheme of the time-resolved transmission set-up excited by nanosecond pulses. 
transmitted by the sample is collimated and focused on entrance slit of a monochromator (CVI CM110 1/8m). The monochromator is set to transmit light centered at $770 \mathrm{~nm}$ where the change in absorption of the sample induced by the LS and HS state switching is large. It is equipped with a grating (1200 grooves $/ \mathrm{mm})$ blazed at $600 \mathrm{~nm}$ and $600 \mu \mathrm{m}$ input and output slits. The output of the monochromator is focused on a fast photodiode connected to a numerical oscilloscope. The transmission signals are averaged over about 120 shots.

\section{Nanosecond photoswitching set-up}

Figure 1b displays the optical setup used to record the time-resolved photoswitching of the samples at the nanosecond time scale. The sample kept in a thermally regulated oven is excited by nanosecond pulses provided by an optical parametric oscillator (Continuum OPO Panther EX). The central wavelength of these pulses is tunable from $410 \mathrm{~nm}$ to $2700 \mathrm{~nm}$. The pulse duration and energy are about $\sim 6 \mathrm{~ns}$ and $40 \mathrm{~mJ}$, respectively. These pulses are slightly focused on the sample on a beam spot of $\Phi \sim 1.5 \mathrm{~mm}$ in diameter. The transmission of the sample is probed at normal incidence, using the Thorlabs CW laser diode (FPL785S). The probe beam transmitted by the sample is collimated and focused on the entrance slit of a monochromator (CVI CM110). The signal at the output of the monochromator is further filtered by a laser-line filter (Thorlabs), collected by a high-speed silicon photodiode and then recorded on a $1 \mathrm{GHz}$ numerical oscilloscope (Tektronik) which triggered the nanosecond pump pulses. The transmission signals are averaged over about 120 shots.

\section{Sample preparation}

The synthesis method of the SCONP and the hybrid Au@SCO has been discussed elsewhere. ${ }^{37}$ The AU@SCO as prepared have high filling factor: about $90 \%$ of the SCONP contain at least one gold nanorod. As shown in the transmission electron microscopy (TEM) image (Fig. 2a), the average size of $\mathrm{Au} @ \mathrm{SCO}$ along the longitudinal and transverse axis is $212 \pm 30 \mathrm{~nm}$ by $106 \pm 24 \mathrm{~nm}$. The embedded AuNRs have an average dimension of $60 \pm 8 \mathrm{~nm}$ 

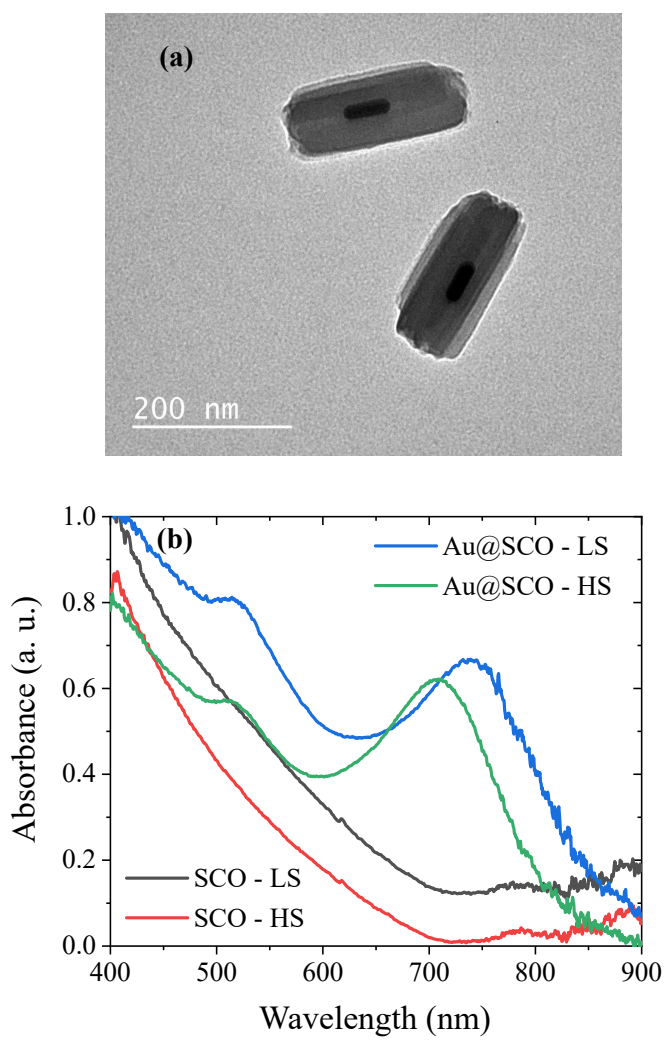

Figure 2: (a) Transmission electron microscopy (TEM) image of Au@SCO (b) Absorbance spectra of SCO and Au@SCO in their low spin (LS) and high spin (HS) states 
in length and $15 \pm 4 \mathrm{~nm}$ in width.

The samples used to record the photoswitching kinetics are prepared as follows. A powder of either SCONP or Au@SCO is dispersed in ethanol, drop-casted, and then dried on a microscope glass slide. The thickness $l_{s}=4.0 \pm 2.0 \mu \mathrm{m}$ of the sample is measured over a 100 $\mu \mathrm{m} * 100 \mu \mathrm{m}$ area with an AFM in tapping mode and averaged. The dried sample is then covered by an other microscope glass slide and inserted in an oven that temperature can be finely controlled $\left( \pm 0.1^{\circ} \mathrm{C}\right)$ from $300 \mathrm{~K}$ to $490 \mathrm{~K}$.
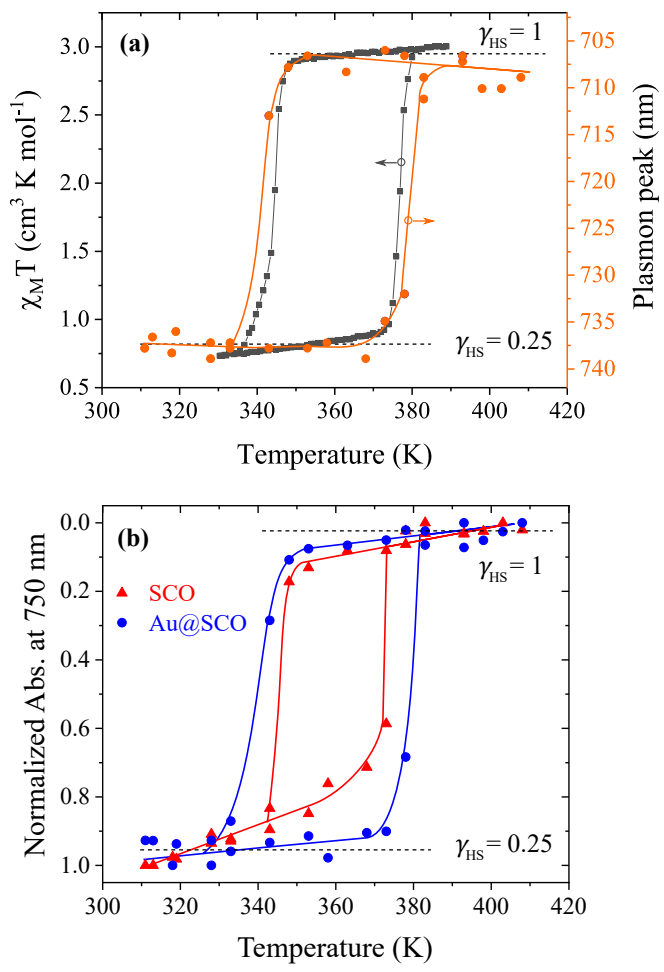

Figure 3: (a) Evolution of the product of $\chi_{M} T$ measured using a SQUID magnetometer (gray square) versus the position of longitudinal plasmon absorption peak (orange circle) within the thermal hysteresis loop of Au@SCO. The solid lines are guidelines. (b) Thermal hysteresis loop of SCO (red triangle) and Au@SCO (blue circle) based on the normalized absorbance at $750 \mathrm{~nm}$. The solid lines are guidelines. 


\section{Results and discussions}

\section{Absorption spectra of the samples in the visible and near infrared.}

Figure $2 \mathrm{~b}$ displays the absorbance of the samples in the LS and HS states in the $400 \mathrm{~nm}$ to $850 \mathrm{~nm}$ spectral range. As its temperature increases the SCONP sample switches from the LS to the HS state and its color turns from pink $(\mathrm{T} \sim 300 \mathrm{~K})$ to white $(\mathrm{T} \sim 410 \mathrm{~K})$. The change in color of SCONP results in a decrease of their absorbance in the HS state. The absorbance spectra of the Au@SCO exhibits two broad peaks, related to the transverse ( $\sim 520 \mathrm{~nm}$ ) and longitudinal (in between $600 \mathrm{~nm}$ and $850 \mathrm{~nm}$ ) surface plasmon modes of the AuNRs. The latter is more marked and its maximum shifts from $\sim 750 \mathrm{~nm}$ to $\sim 710 \mathrm{~nm}$ when the temperature is increased from $300 \mathrm{~K}$ to $410 \mathrm{~K}$. Comparison between the magnetic and absorption measurements (Fig. 3a) indicates this shift of the longitudinal SPR is associated to the switching from the LS to the HS state of the spin crossover shell within the Au@SCO. Figure $3 \mathrm{~b}$ also indicates the switching from the LS to HS state results in a decrease of the normalized absorbance of both SCO and Au@SCO samples at $750 \mathrm{~nm}$.

\section{Study of the photoswitching at the millisecond time scale}

\section{Experimental results}

Figure 4a shows the temporal evolution transmission upon QCW excitation of $\mathrm{Au} @ \mathrm{SCO}$ (blue dots) and SCONP (red dots) samples by an optical pulse of power and duration of $I_{p}=270 \mathrm{~mW}$ and $t_{p}=490 \mathrm{~ms}$, respectively.

Upon excitation, the increase in transmission is fitted using a simple mono-exponential model $S(t)=S_{0}\left(1-\exp \left(-t / \tau_{c}\right)\right)$, where the rate of change is expressed by the characteristic time $\tau_{c}$. The fitted values of $\tau_{c}$ are $60 \mathrm{~ms}$ and $300 \mathrm{~ms}$ for Au@SCO and SCONP, respectively. After the pulse excitation, the transmission of the samples remains constant and it does not relax back to its initial value. To recover its initial transmission, the sample has to be cooled down below $330 \mathrm{~K}$. In Figure 4a, we have also estimated the ratio of converted NPs during the 

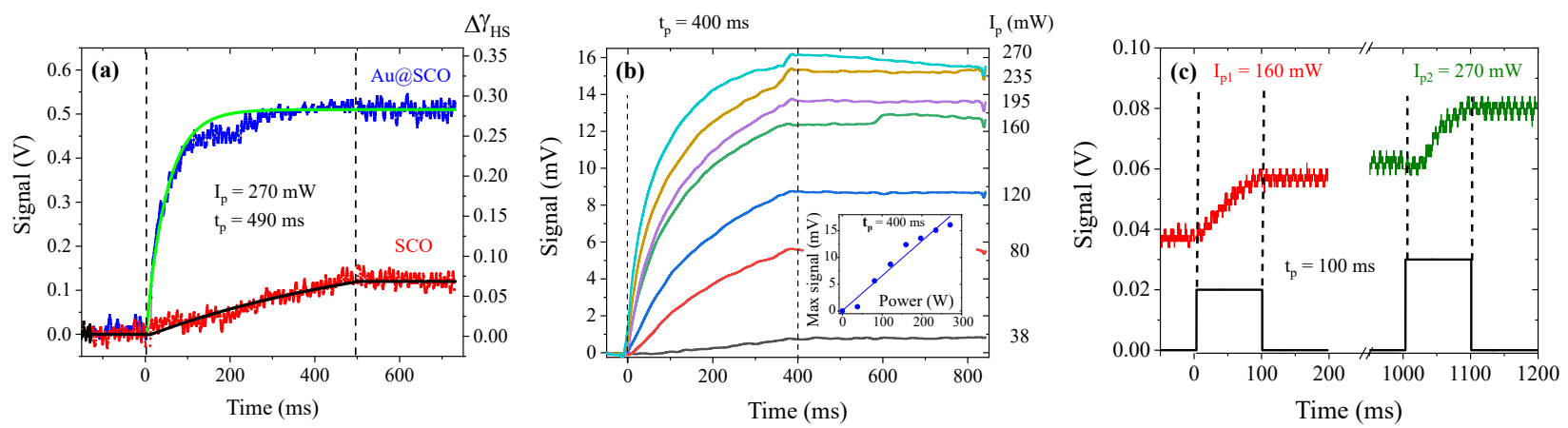

Figure 4: (a) Evolution of the transmitted signal during the photoswitching of $\mathrm{Au} @ \mathrm{SCO}$ (blue dots) and SCONP (red dots) induced at $T=370 \mathrm{~K}$ by an optical pulse that peak power and pulse duration are $I_{p}=270 \mathrm{~mW}$ and $t_{p}=490 \mathrm{~ms}$. The dashed lines indicate the start and end of the pump pulse. The solid lines are mono-exponential fits considering the transmission writes $S(t)=S_{0}\left(1-\exp \left(-t / \tau_{c}\right)\right)$, where $\tau_{c}$ are $60 \mathrm{~ms}$ and $300 \mathrm{~ms}$ for Au@SCO and SCONP, respectively. (b) Evolution of the transmitted signal of the Au@SCO sample upon excitation by a $400 \mathrm{~ms}$ optical pulse at various peak powers $I_{p}$. The inset evidences the linear increase of the maximum signal on the pump power. (c) Step photoswitching in Au@SCO induced by two consecutive 100 ms pump pulses with different peak powers.

photoswitching experiment (Fig. 4b). The way we estimated the change in the HS fraction $\Delta \gamma_{H S}$ is as follows. Before exciting the sample, we recorded the change in amplitude $\Delta \mathrm{V}$ of the signal recorded by the photodiode at the exit of the monochromator when the sample is set at $\mathrm{T}=300 \mathrm{~K}$ and at $\mathrm{T}=430 \mathrm{~K}$ in the $\mathrm{HS}$ state. We consider this change in amplitude $\Delta V$ is associated to $\Delta \gamma_{H S}=1$. Then we consider that upon laser excitation the change in $\Delta \gamma_{H S}(t)$ scales with respect to the signal $\mathrm{V}(\mathrm{t})$ recorded by the photodiode: $\Delta \gamma_{H S}(t)=\frac{\Delta V(t)}{\Delta V}$. Accordingly, we estimate that a QCW optical pulse of $I_{p}=270 \mathrm{~mW}$ switches about $28 \%$ $\left(\Delta \gamma_{H S}=0.28\right)$ of Au@SCO NPs from LS to HS states while it is less than $10 \%$ for SCONP. Regarding the photoswitching time, the conversion is achieved within 250 ms for Au@SCO when the pulse duration is $t_{p}=490 \mathrm{~ms}$. Note that when the pump pulse ends the slope of the signal is almost zero indicating we reached a steady state regime. On the contrary, upon excitation the SCONP sample does not reach such a steady state even after $490 \mathrm{~ms}$.

We also recorded transmission of the Au@SCO upon excitation by a $1 \mathrm{~Hz}$ pump train (Fig. 4c). The exciting pulses have the same peak power $\left(I_{p 1}=160 \mathrm{~mW}\right)$ and duration $\left(t_{p}=100 \mathrm{~ms}\right)$. Note that only the first pulse induces a change in transmission (red curve in 
Fig. 4c). When another pulse excites the sample, the transmission does not further change. To further increase the transmission of the sample, one has to increase temperature of the sample or laser peak power as illustrated in Fig. 4c where pump peak power was increased from $I_{p 1}=160 \mathrm{~mW}$ to $I_{p 2}=270 \mathrm{~mW}$.

Finally, to evidence the impact of the change in $\Delta \gamma_{H S}$ on the photoswitching kinetics, we normalize the transmission of the sample under different pulse powers for given pulse duration. One can notice that for a given pulse duration, the higher the peak power density,
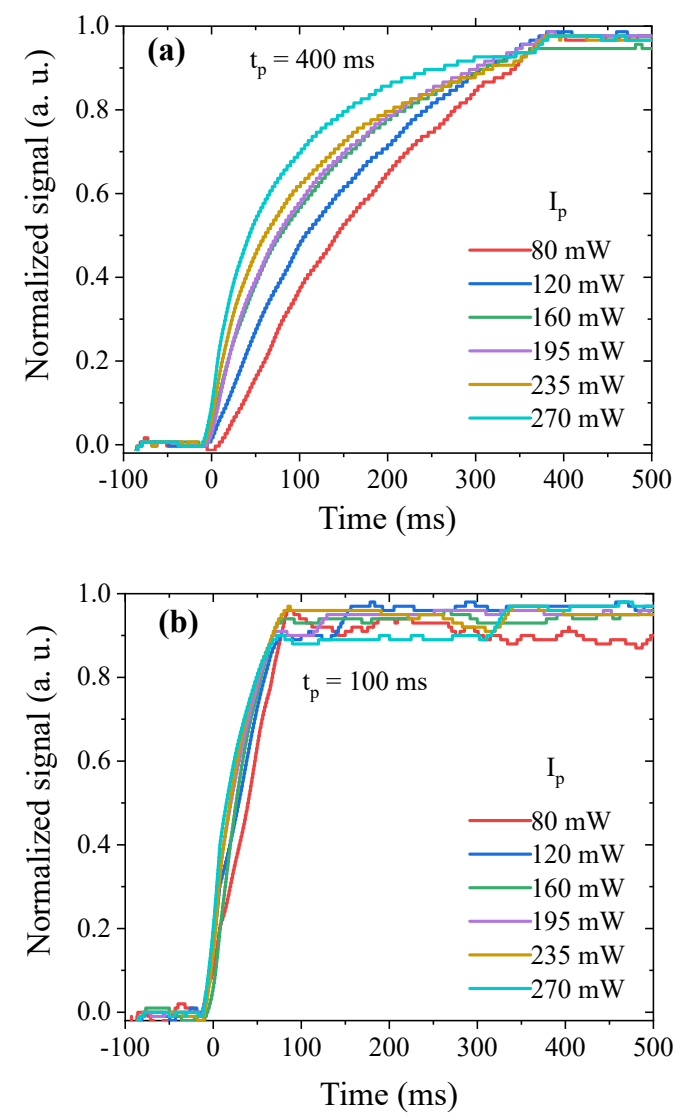

Figure 5: Normalized transmission signal over the pulse duration of (a) $t_{p}=400 \mathrm{~ms}$ and (b) $t_{p}=100 \mathrm{~ms}$.

the faster the photoswitching (Fig. 5a). Besides for short pulse duration $t_{p}=100 \mathrm{~ms}$, the kinetics remains almost unchanged whatever the pulse power (Fig. 5b) while a noticeable change is observed for longer pulse duration (Fig. 5a). 


\section{Discussion}

Hereafter, we will assume that upon QWC excitation the change in temperature can result almost instantaneously in an increase of $\Delta \gamma_{H S}$ in both the Au@SCO and SCONP samples. In our QCW experiments, the change in $\Delta \gamma_{H S}$ versus $\Delta T$ can be estimated from Fig.3. Note that in the latter figure at the rising edge of the hysteresis loop $(\mathrm{T} \sim 370 \mathrm{~K}), \gamma_{H S}$ (and therefore $\left.\Delta \gamma_{H S}\right)$ scales almost linearly with $\Delta \mathrm{T}$ along the ascending branch of the thermal hysteresis. Hence to model our experimental data one has to account for the temperature evolution within our sample upon laser excitation.

Upon QCW pulse excitation, the change in temperature of the sample $\Delta T$ is governed by the heat equation that writes:

$$
\frac{\partial \Delta T}{\partial t}=D_{t h} \nabla^{2}(\Delta T)+\frac{Q}{\rho C_{p}}
$$

where $D_{t h}, \rho, C_{p}$ and $Q$ are heat diffusivity, initial mass density, heat capacity and the heat deposited in the sample, respectively. The latter equation is valid for a homogeneous solid whose thermal conductivity is independent of the temperature. For QCW pulse excitation $Q=\alpha\left(\lambda_{p}\right) I_{p} / V$ where $\alpha\left(\lambda_{p}\right), I_{p}$ and $V$ are the absorption coefficient of the sample at a pump wavelength $\lambda_{p}$, the power density of optical pump pulse and the excited volume, respectively. The resolution of Eq. 1 along with the boundaries, initial conditions and used main approximations are given in the supplementary material. Under our experimental conditions where the thickness $l_{s}$ of the sample is small compared to the diameter $(\mathrm{d}=2 \mathrm{a})$ of the photo-excited area, the temperature at the center of our very thin sample writes:

$$
\Delta T_{0} \approx \frac{A_{0} l_{s}^{2}}{8 D_{t h}}
$$

where $A_{0}=\alpha\left(\lambda_{p}\right) I_{p} /\left(V \rho C_{p}\right)$. The mean temperature of the sample at steady state writes:

$$
\Delta T_{m} \approx \frac{2}{3} \Delta T_{0}
$$


Since $\Delta T_{m} \propto \Delta T_{0} \propto I_{p}$, the mean temperature of the sample increases linearly with the power of the exciting laser. One can also evaluate the kinetic for temperature rise. The latter writes:

$$
\Delta T(r, z, t) \approx \Delta T(r, z)\left[1-\exp \left(-\frac{t}{\tau_{c, z}}\right)\right] \times\left[1-\exp \left(-\frac{t}{\tau_{c, r}}\right)\right]
$$

where the time constants $\tau_{c, z}=\frac{1}{D_{t h}}\left(\frac{l^{2}}{\pi^{2}}\right)$ and $\tau_{c, r} \approx \frac{1}{D_{t h}}\left(\frac{a}{2.4}\right)^{2}$ account for diffusion of heat along the thickness and diameter of the sample, respectively. According to Eq. 2-4 and knowing the radius $a$ of the beam spot of the pump pulse, the thickness $l_{s}$, the thermal diffusivity $D_{t h}$, the density $\rho$ and the specific heat $C_{p}$ of the sample, one can compute the temperature at the center of the disk $\Delta T_{0}$ (Eq. 2), the mean temperature at steady state $\Delta T_{m}$ (Eq. 3) and the time constant. The heat capacity of the $\left[\mathrm{Fe}(\mathrm{Htrz})_{2} \operatorname{trz}\right]\left(\mathrm{BF}_{4}\right)$ is $C_{p} \sim 1 \mathrm{~J} \cdot \mathrm{g}^{-1} \cdot \mathrm{K}^{-1} .{ }^{39}$ The bulk thermal diffusivity of this material has not been reported, but we will consider that it behaves like a conventional polymer with $D_{t h} \sim 1.2 \times 10^{-7} \mathrm{~m}^{2} / \mathrm{s}$. The density of the $\left[\mathrm{Fe}(\mathrm{Htrz})_{2} \operatorname{trz}\right]\left(\mathrm{BF}_{4}\right)$ is $\rho \sim 1.8 \mathrm{~g} / \mathrm{cm}^{3}{ }^{39}$ However, the technique used to prepare our samples makes the SCO NPs randomly packed. Their actual density is therefore lesser because it depends on the way the different nanorods are arranged in the powder. It has been shown that the occupied volume fraction scales at most like $\phi \approx \frac{D}{L}$ where $D$ and $L$ are the diameter and length of the rods, respectively. Since for our sample $\frac{D}{L} \approx 0.5$ we estimate $\rho \sim 0.9 \mathrm{~g} / \mathrm{cm}^{3} .{ }^{40}$ Knowing these parameters, we can readily compute the steady state temperature in the center of the SCONP and Au@SCO samples upon excitation by a QCW pump pulse. In these computations, the main difference between SCONP and $\mathrm{Au} @ \mathrm{SCO}$ samples is their absorption $\alpha\left(\lambda_{p}\right)$ at the pump wavelength. We will consider that $\sim 60 \%$ and $\sim 20 \%$ of the pump power is absorbed by the Au@SCO and SCONP sample, respectively. Accordingly, considering the pump power $I_{p}=160 \mathrm{~mW}$ is focused on beam spot of $\Phi \sim 0.8 \mathrm{~mm}$, one finds $\Delta T_{0}(\mathrm{Au} @ \mathrm{SCO}) \sim 1.38 \mathrm{~K}$ and $\Delta T_{m}(\mathrm{Au} @ \mathrm{SCO}) \sim 0.92 \mathrm{~K}$. whereas for SCONP $\Delta T_{0}(\mathrm{SCONP}) \sim 0.45 \mathrm{~K}$ and $\Delta T_{m}(\mathrm{SCONP}) \sim 0.3 \mathrm{~K}$. 
When the peak power of the pump is increased from $160 \mathrm{~mW}$ to $270 \mathrm{~mW}: \Delta T_{m}(\mathrm{Au} @ \mathrm{SCO}) \sim$ $1.55 \mathrm{~K}$ and $\Delta T_{m}(\mathrm{SCONP}) \sim 0.52 \mathrm{~K}$. One can notice that in agreement with Fig. $4 \mathrm{~b}$, the latter peak power gives rise to a measurable change in transmission for both SCONP and Au@SCO samples.

Finally, we evaluate the characteristic time $\tau_{c}$ necessary for the sample to be at a quasisteady temperature. The characteristic times related to diffusion of heat along the thickness $l_{s} \sim 4 \mu \mathrm{m}$ and radius $r \sim 400 \mu \mathrm{m}$ of the sample are $\tau_{c, z} \approx 13 \mu \mathrm{s}$ and $\tau_{c, r} \approx 230 \mathrm{~ms}$, respectively. Since $\tau_{c, r} \gg \tau_{c, z}$, Eq. 4 indicates that for our samples the rise in temperature is mainly governed by the time constant $\tau_{c, r}$. The value of $\tau_{c, r}$ compares well with the fitted time constant $\tau_{c} \sim 160 \mathrm{~ms}$ (Fig. 4a). All these simple computations stress that the phenomena displayed in Fig. 4a-b and Fig. 5a-b are mainly due to the rise in temperature of the sample upon laser excitation and that their kinetics is mainly related to diffusion of heat along the diameter of the pump beam spot.

These computations, which agree well with the experimental data, also underline that indeed the changes in transmission of the samples upon their photo-excitation are solely due to the increase of $\gamma_{H S}$ upon increase in temperature. This allows us to propose a scenario that accounts for the behaviors displayed in Fig. 4. As illustrated in Fig. 6, the sample is initially set at the temperature $T_{0}$ (point A) just below the ascending branch of the thermal hysteresis loop in the phase diagram of $\left(T, \gamma_{H S}\right)$. Upon excitation by a QCW laser pulse, the temperature of the sample rapidly increases. The change in temperature results in an increase of $\gamma_{H S}$. Hence, the sample is brought at a higher $\gamma_{H S}$ position along the thermal hysteresis loop. When the pump QCW pulse ends, the sample is set at point B. The value of $\gamma_{H S}$ along the thermal hysteresis loop is fixed by the mean temperature that the sample reaches when the pump pulse ends $T_{B}=T_{0}+\Delta T_{m}\left(I_{p 1}, T_{1}\right)$. The rate of growth of the HS state is governed by the kinetics of heat diffusion within the sample. After excitation, the sample cools down back to its initial temperature and it is set at point C. Notice that further excitation of the sample with a new QCW pulse that has exactly the same peak power and 

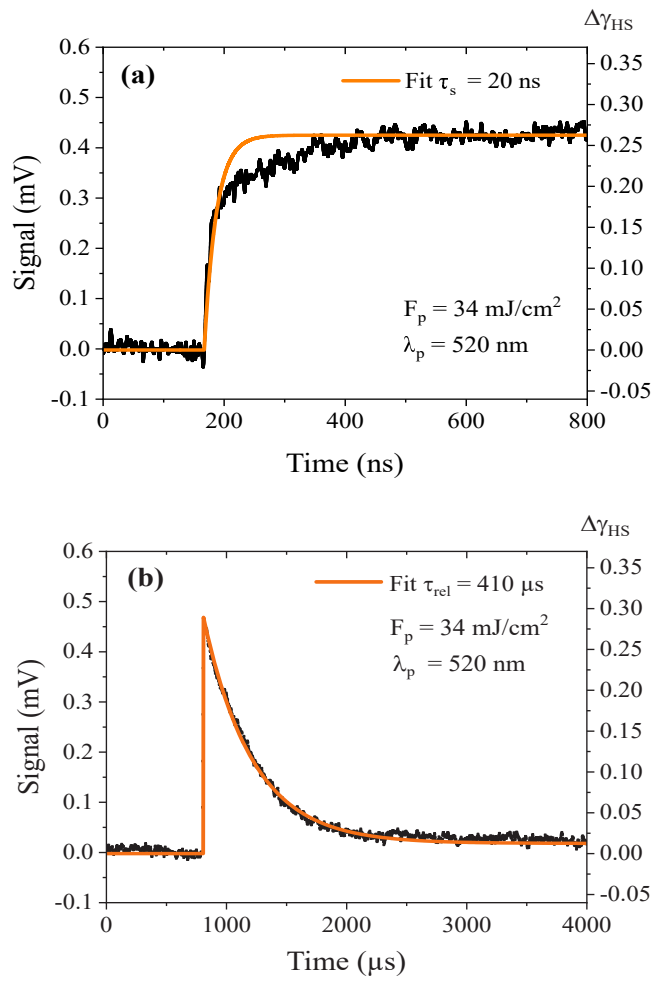

Figure 6: The evolution of HS fraction induced by QCW pulsed excitation is illustrated in the $\left(T, \gamma_{H S}\right)$ hysteresis loop of the sample. The HS fraction can be further increased by a second more energetic optical pulse which brings the system to a higher temperature $\left(\Delta T_{2}>\Delta T_{1}\right)$. 
duration will bring the sample back to point B. However, if the peak power is increased $\left(I_{p_{2}}>I_{p_{1}}\right)$, the sample will be brought at a higher temperature $T=T_{0}+\Delta T_{2}$ that will further increase $\gamma_{H S}$ (point D). Then the sample will cool down (point E). Of course further increase of $\gamma_{H S}$ can be achieved increasing the pump peak power. This process stops when $\gamma_{H S}=1$. The described phenomenon accounts for all the observations reported in Fig. 4c.

It is worth noticing that the spin state transition responsible for the increase $\gamma_{H S}$ results in a modification of the absorption of the sample $\alpha\left(\lambda_{p}\right)$ as well as most of the thermodynamic constants of the sample such as $\rho, C_{p}, D_{t h}$. Hence the computation we performed holds only if the change of $\gamma_{H S}$ is small and lower than $\sim 0.1$. For larger increase in $\gamma_{H S}$, the photoswitching kinetics is expected to be modified. This likely accounts for the change in kinetics displayed in Fig.5a.

\section{Study of the photoswitching at the nanosecond time scale}

\section{Experimental results}

Figure 7 displays a typical evolution of the transmission signal $S(t)$ of the Au@SCO sample initially set at $T_{0}=333 \mathrm{~K}$ upon excitation by a nanosecond laser pulse centered at $520 \mathrm{~nm}$ that fluence is $F_{p} \sim 34 \mathrm{~mJ} / \mathrm{cm}^{2}$. The evolution of $S(t)$ indicates the transmission of the sample increases rapidly within few tens of nanoseconds and remains constant for $\sim 20 \mu \mathrm{s}$. It then slowly relaxes within few milliseconds (Fig. 7b). Using the following function $S(t)=$

$S_{0}\left(1-\exp \left(-t / \tau_{s}\right)\right)$ where $\tau_{s}$ is the switching time constant, we are able to fit the rapid increase of $S(t)$ considering $\tau_{s}=20 \pm 2$ ns (Fig. 7b). Similarly, we are able to nicely fit the relaxation of $S(t)$ considering a mono-exponential decay law with a time constant $\tau_{\text {rel }}=410 \pm 10 \mu \mathrm{s}$ (Fig. 7c).

This experiment was also performed for four different lesser laser fluences (Fig. 8a). The increase in transmission is well fitted using the same switching time of $\tau_{s}=300 \pm 80 \mathrm{~ns}$ (Fig. 8b). Similarly, the relaxation is also well fitted using the same relaxation time of $\tau_{\text {rel }}=60 \pm 6 \mu$ s regardless of the excitation fluence (Fig. 8c). 


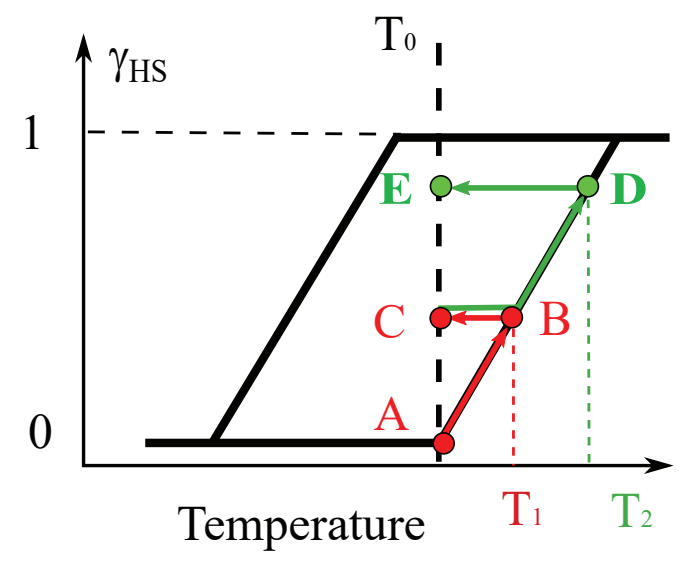

Figure 7: (a) Evolution of the transmitted signal $\mathrm{S}(\mathrm{t})$ of $\mathrm{Au} @ \mathrm{SCO}$ sample set at $T_{0}=333 \mathrm{~K}$ upon excitation by $6 \mathrm{~ns}$ pulse which central frequency is $520 \mathrm{~nm}$ and fluence $34 \mathrm{~mJ} / \mathrm{cm}^{2}$. The orange line stands for the mono-exponential fit which writes $S(t)=S_{0}\left(1-\exp \left(-t / \tau_{s}\right)\right)$. (b) Relaxation of the transmitted signal $S(t)$ at microsecond time scale. The orange line stands for the mono-exponential fit which writes $\left.S(t)=S_{0} \exp \left(-t / \tau_{\text {rel }}\right)\right)$.
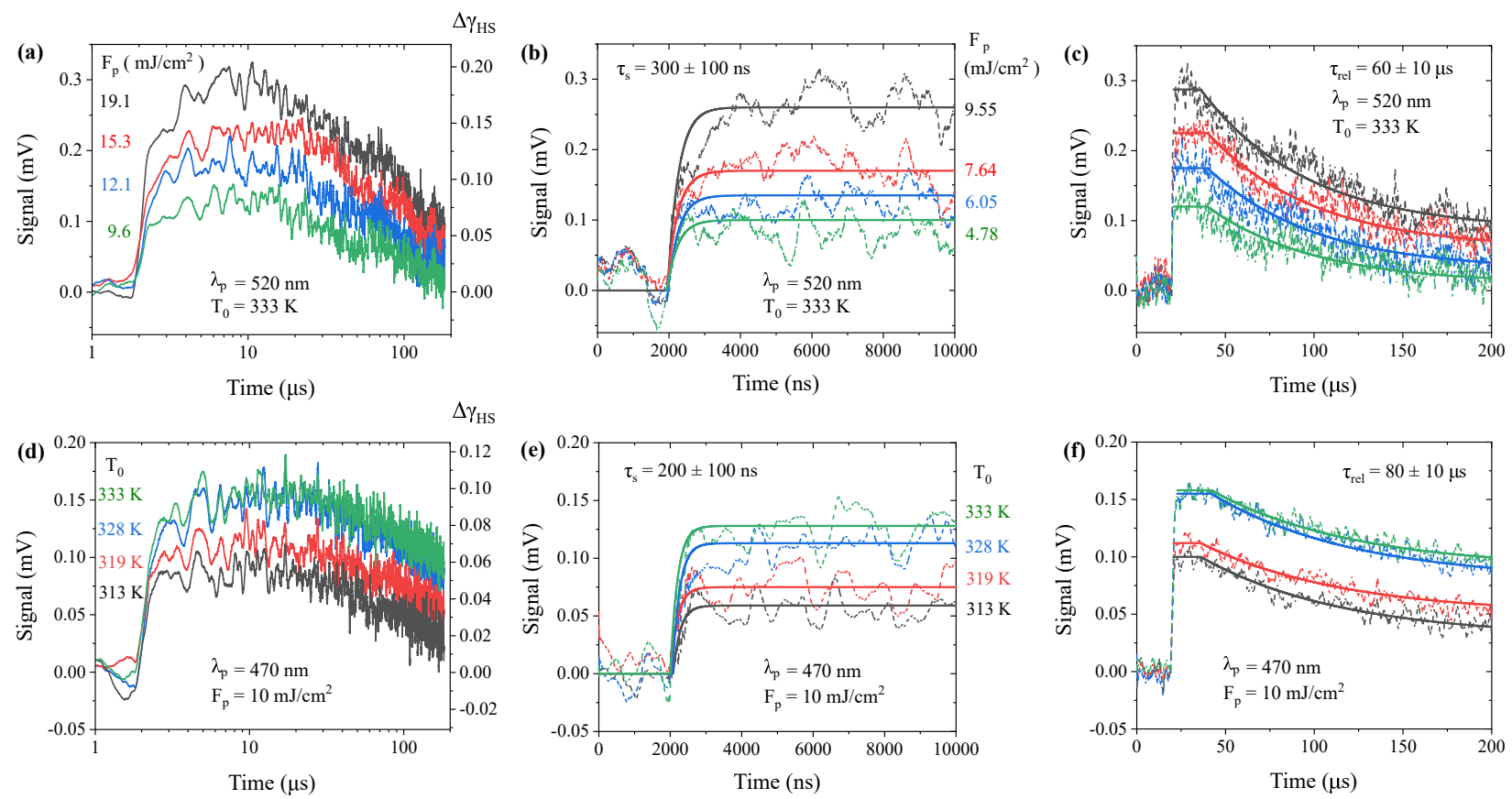

Figure 8: (a-b-c) Temporal evolution of transmission in Au@SCO sample at initial temperature $T_{0}=333 \mathrm{~K}$ excited by a $520 \mathrm{~nm}$ optical pulse at different pump fluence from $9.6 \mathrm{~mJ} / \mathrm{cm}^{2}$ to $19.1 \mathrm{~mJ} / \mathrm{cm}^{2}$. Mono-exponential fits show characteristic rise time $\tau_{s}=300 \pm 100 \mathrm{~ns}$ and relaxation time $\tau_{\text {rel }}=60 \pm 10 \mu$ s independent of pump fluence. (d-e-f) Temporal evolution of transmission in Au@SCO sample at different initial temperature $T_{0}$ ranging from $313 \mathrm{~K}$ to $333 \mathrm{~K}$, excited by a $470 \mathrm{~nm}$ optical pulse at fluence of $10 \mathrm{~mJ} / \mathrm{cm}^{2}$. Mono-exponential fits of the characteristic rise time $\tau_{s}=200 \pm 100 \mathrm{~ns}$ and relaxation time $\tau_{r e l}=80 \pm 10 \mu \mathrm{s}$ which are independent of initial temperature $T_{0}$. 
The sample is also excited using nanosecond laser pulses centered at $470 \mathrm{~nm}$ (Fig. 8d-e-f). However in this case, we vary the initial temperature while keeping the laser fluence constant. The Figure 8d displays the evolution of the transmission signal recorded at constant pump fluence $F_{p}=10 \mathrm{~mJ} / \mathrm{cm}^{2}$ and different initial temperatures ranging from $313 \mathrm{~K}$ to $333 \mathrm{~K}$. The amplitude of the signal decreases as the initial temperature is decreased. We are able to fit the rising and relaxation times of the signal applying mono-exponential functions. Here again whatever the initial temperature of the sample, we find that the characteristic switching time is $\tau_{s}=200 \pm 50 \mathrm{~ns}$ (Fig. 8e), whereas the relaxation time is $\tau_{r e l}=80 \pm 8 \mu \mathrm{s}$ (Fig. 8f).

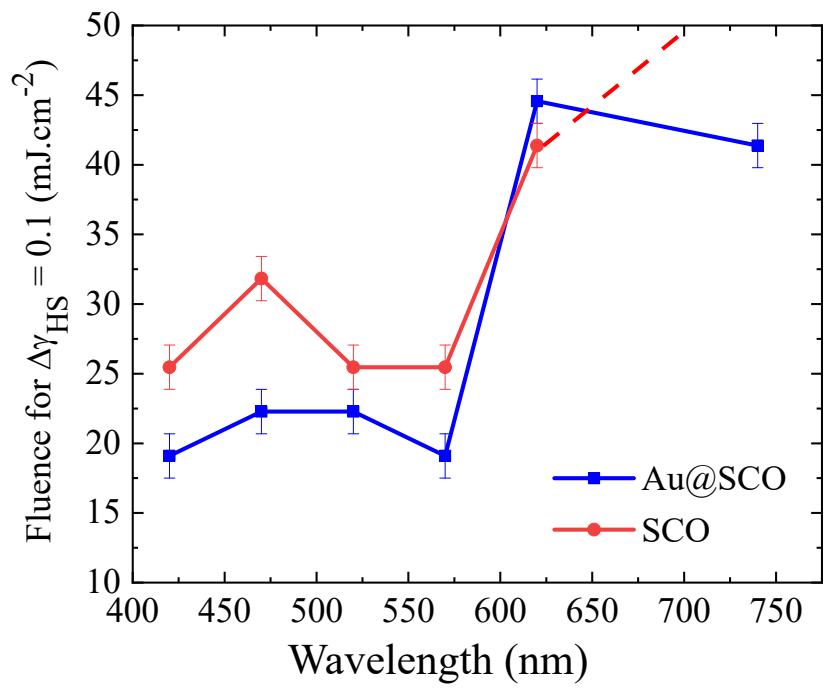

Figure 9: The wavelength dependence of the pump fluence threshold required to achieve $\Delta \gamma_{H S}=0.1$ in Au@SCO and SCO samples.

Finally, we tune the central wavelength of the nanosecond pulse and record the laser fluence threshold needed to increase the transmission of the sample by $10 \%\left(\Delta \gamma_{H S}=0.1\right.$. As displayed in Fig. 9, the threshold fluence remains almost constant in the $420 \mathrm{~nm}$ to $570 \mathrm{~nm}$ spectral range and is about $F_{t h} \sim 28 \pm 2 \mathrm{~mJ} / \mathrm{cm}^{2}$ and $F_{t h} \sim 22 \pm 2 \mathrm{~mJ} / \mathrm{cm}^{2}$ for SCONP and $\mathrm{Au} @ \mathrm{SCO}$ sample, respectively. It rapidly increases at longer wavelengths. Above $700 \mathrm{~nm}$, the laser fluence required to record such change in transmission is too high and always damage the SCONP sample. Regarding Au@SCO at $620 \mathrm{~nm}$, the laser fluence threshold is $F_{t h} \sim 44 \pm 2 \mathrm{~mJ} / \mathrm{cm}^{2}$ and slightly drops towards $42 \pm 2 \mathrm{~mJ} / \mathrm{cm}^{2}$ as one set the pump wavelength at $750 \mathrm{~nm}$ close to the plasmon resonance of the Au@SCO. 


\section{Discussion}

Equation (1) also describes the photoswitching upon excitation of the sample by nanosecond pulses. However Fig. 7a reveals that photoswitching of the nanohybrids takes place on a time scale $\sim 20 \mathrm{~ns}$ that is about three orders of magnitude smaller that $\sim 13 \mu$ s the time it takes for heat to diffuse within our $4 \mu \mathrm{m}$ thick sample. In fact, in the time $t \sim 20 \mathrm{~ns}$ the heat diffuses over a length $L_{c}=2 \pi \sqrt{D_{t h} T} \sim 310 \mathrm{~nm}$ that is about the characteristic length of our NPs. This indicates, we can neglect the diffusion of heat in between the NPs during the first tens of nanoseconds. Neglecting the heat diffusion in Eq.(1), the rise in temperature $\Delta T$ upon excitation of the sample by a nanosecond pulse writes:

$$
\Delta T=\frac{Q}{\rho C_{p}}
$$

where $\mathrm{Q}$ is the generated heat. The latter writes $Q=\frac{\alpha\left(\lambda_{p}\right) E_{p}}{e}$ where $\alpha\left(\lambda_{p}\right), E_{p}$ and $e$ are absorption of the sample at the pump wavelength, the pump laser fluence and the thickness of the sample, respectively. ${ }^{30}$ Contrary to the QCW pulse excitation (see Eq.(2)), $\Delta T$ does not depend on the thermal diffusivity. Besides, at variance with the approximations used to analyze QCW pulse excitation, all the particles, even those lying on the top and bottom surfaces, are heated.

To evaluate the rise in temperature $\Delta T$ of the Au@SCO sample displayed in Fig. 7, we will here again consider this sample has the following characteristic parameters: $\rho \sim 0.9 \mathrm{~g} / \mathrm{cm}^{3}$, $C_{p} \sim 1 \mathrm{~J} \cdot \mathrm{g}^{-1} \cdot \mathrm{K}^{-1}$. Since at $\lambda_{p}=520 \mathrm{~nm}, \sim 60 \%$ of the pump fluence $F_{p}=34 \mathrm{~mJ} / \mathrm{cm}^{2}$ is absorbed within the sample thickness of $e \sim 4 \mu \mathrm{m}$ when $\Delta T \sim 56 \mathrm{~K}$. If the initial temperature of the sample is $T_{0}=333 \mathrm{~K}$, close to the end of the descending branch of the thermal hysteresis loop, upon excitation its temperature becomes $T=T_{0}+\Delta T \sim 389 \mathrm{~K}$ and the sample is set $\sim 10 \mathrm{~K}$ above the ascending branch of the thermal hysteresis loop (see Fig.3). Therefore, the HS fraction rapidly grows within each NPs. This accounts for the $\tau_{s} \sim 20 \mathrm{~ns}$ nanosecond photo-switching time. This switching time likely depends on the size 
of the NPs. ${ }^{33}$

We have also evaluated the increase in temperature upon excitation of the sample in the experiment displayed in Fig. 8a. Before excitation, the temperature of sample is set at $T_{0}=333 \mathrm{~K}$. The computed rise in temperature $\Delta T$ is $\sim 9.3 \mathrm{~K}, 12 \mathrm{~K}, 14.5 \mathrm{~K}$ and $18.5 \mathrm{~K}$ for laser fluence of $9.6 \mathrm{~mJ} / \mathrm{cm}^{2}, 12 \mathrm{~mJ} / \mathrm{cm}^{2}, 15 \mathrm{~mJ} / \mathrm{cm}^{2}$ and $19 \mathrm{~mJ} / \mathrm{cm}^{2}$, respectively. Since the temperature of sample $T=T_{0}+\Delta T$ is always $\leq 352 \mathrm{~K}$, it should remain below the ascending branch of the thermal hysteresis (see Fig.3). Therefore, it is surprising to record a photoswitching of a non-negligible amount of NPs. However, the simple computation we performed neglects the attenuation of the pump pulse during its propagation within the sample. In fact, as the pulse propagates within the sample, it drops less and less energy. The increase of temperature is therefore not homogeneous along the sample thickness. It is more important at the top of the sample and likely above $30 \mathrm{~K}$. Besides, the increase and saturation of the HS fraction happen on time scale of about $\tau_{s} \sim 300 \mathrm{~ns}$. This time scale is about ten times larger than the time it takes for heat to diffuse within a single NP ( $\sim 20 \mathrm{~ns})$. During $300 \mathrm{~ns}$ heat diffuses over a characteristic length $L_{d}=2 \pi \sqrt{D_{t h} \tau_{s}} \sim 1 \mu \mathrm{m}$ which is large compared to the size of the Au@SCO. Therefore, while during the first tens of nanoseconds, it is mostly the NPs at the top of the sample that are photoswitched, as time goes on and thermal diffusion takes place, the temperature of NPs beneath the top surface further increases and they are brought along the ascending branch of the thermal hysteresis loop giving rise to an increase of the HS fraction. Afterwards the heat generated within the NPs further diffuses within the whole sample.

As previously mentioned, the characteristic time for thermalization of our $4 \mu \mathrm{m}$ thick sample is $\tau_{c, z} \sim 13 \mu \mathrm{s}$. After this characteristic time, we do not expect to record any further increase of the HS fraction. This latter behavior is indeed in good agreement with the time scale of the plateau $(\sim 20 \mu \mathrm{s})$ displayed in Fig. 7a, Fig. 8c-f. At longer time scale, relaxation from the HS to the LS spin state should proceed and the higher $\Delta \gamma_{H S}$ the longer the relaxation time. Here again, this agrees with the data displayed in Fig. 7-8. 
As we noticed above, the initial temperature of the sample matters. By keeping the laser fluence constant while reducing the initial temperature, the sample is moved away from the ascending branch of the thermal hysteresis loop and the amplitude of the transmitted signal is reduced. This is in agreement with the data displayed in Fig. 8d-e-f where a nanosecond pulse centered at $470 \mathrm{~nm}$ is used to excite the sample. At this wavelength the sample is more absorbing. Hence, even if the $E_{p}=10 \mathrm{~mJ} / \mathrm{cm}^{2}$ considering $\sim 85 \%$ of the pump energy is absorbed by the sample, the rise in temperature is $\Delta T \sim 23 \mathrm{~K}$. Therefore upon excitation, the sample is quenched at the edge of ascending branch of the thermal hysteresis loop. Besides, in agreement with the evolution recorded exciting the sample at $520 \mathrm{~nm}$, as the HS switching happens on shorter time scale $\left(\tau_{s} \sim 200 \pm 100 \mathrm{~ns}\right)$, the relaxation is recorded on a smaller time scale $\left(\tau_{\text {rel }} \sim 80 \pm 10 \mu \mathrm{s}\right)$.

The processes described above can take place in both SCONP and Au@SCO samples. The main difference between these samples is their difference in absorption at a given wavelength. The latter directly impacts the temperature at which the sample is quenched upon excitation. At fixed laser fluence $F_{p}$, the higher the absorption the higher the rise in temperature. Conversely, one can also fix the change in transmission and record the evolution pump fluence threshold $F_{t h}\left(\lambda_{p}\right)$ required to induce such change in transmission at a given pump wavelength $\lambda_{p}$. The pump fluence threshold $F_{t h}\left(\lambda_{p}\right)$ scales with the amount of energy absorbed by the sample and is proportional to the absorbance $\mathrm{A}\left(\lambda_{p}\right)$ of the sample at the exciting wavelength. Thanks to the inclusion of Au nanorod in the SCO NPs, whatever the wavelength, absorption of the Au@SCO is larger than the SCONP. Therefore, in agreement with data displayed in Fig. $9, F_{t h}\left(\lambda_{p}\right)$ is larger for the SCONP sample. For the SCONP in the LS state, absorption rapidly decreases in the near IR spectral range, thus $F_{t h}\left(\lambda_{p}\right)$ should also rapidly increase in the visible range, whereas for the $\mathrm{Au} @ \mathrm{SCO}$, it should decrease thanks to the plasmon resonance. While for the SCO the reduction of $F_{t h}$ is lower than expected, these trends are evidenced in Fig. 9.

We have previously shown the evolution of signal recorded upon excitation by a nanosec- 
ond laser pulse is related to the evolution of the position of the sample within its $\left(T, \gamma_{H S}\right)$ phase diagram. ${ }^{30}$ This scenario is depicted in Fig. 10a along with the evolution of transmission signal (Fig. 10b). The sample is initially set below the thermal hysteresis loop $\left(\gamma_{H S} \sim 0\right)$ near the ascending branch of the thermal hysteresis loop (point A). Upon excitation and absorption of the laser light by the sample, the temperature of the sample rapidly increases while most of the SCONP remain in their initial state (Point B). When the fluence is high enough, the SCO sample is over heated and the HS fraction rapidly growths. Then as heat diffuses, the average temperature lowers due to the endothermic nature of the LS to HS transition process. This process stops when the temperature of the sample reached the ascending branch of the thermal hysteresis loop (Point C). Beyond this temperature, the HS state fraction remains constant whereas the temperature further decreases. This lasts as long as the temperature of the sample has not reached the descending branch of the thermal hysteresis loop (Point D). Below that latter temperature both the LS fraction and temperature relax back to their initial position within the $\left(T, \gamma_{H S}\right)$ phase diagram.

\section{General discussion}

The experiments performed on a thin layer of AuSCO and SCONP and the analysis presented above provide a lot of information about laser-induced LS to HS state thermal photoswtiching of an ensemble of NPs we are going to summarize hereafter.

The first important result that our study convey is that insertion of AuNRs in an SCO $\mathrm{NP}$ is an interesting mean to increase the absorption of the Au@SCO in a very broad spectral range. But for a sample made out of an ensemble of $\mathrm{Au} @ \mathrm{SCO}$, it does not guarantee a fast photoswitching or a very large increase in temperature of a sample. These latter depend on parameters which strongly impact the solutions of the heat equation. If one considers heat diffusion within a thin disk that surfaces are kept at room temperature, they are two kinds of parameters. The first ones we will consider as intrinsic parameters are related to the studied material. These are the mass density $\rho$, the heat capacity $C_{p}$, the thermal diffusivity $D_{t h}$, 

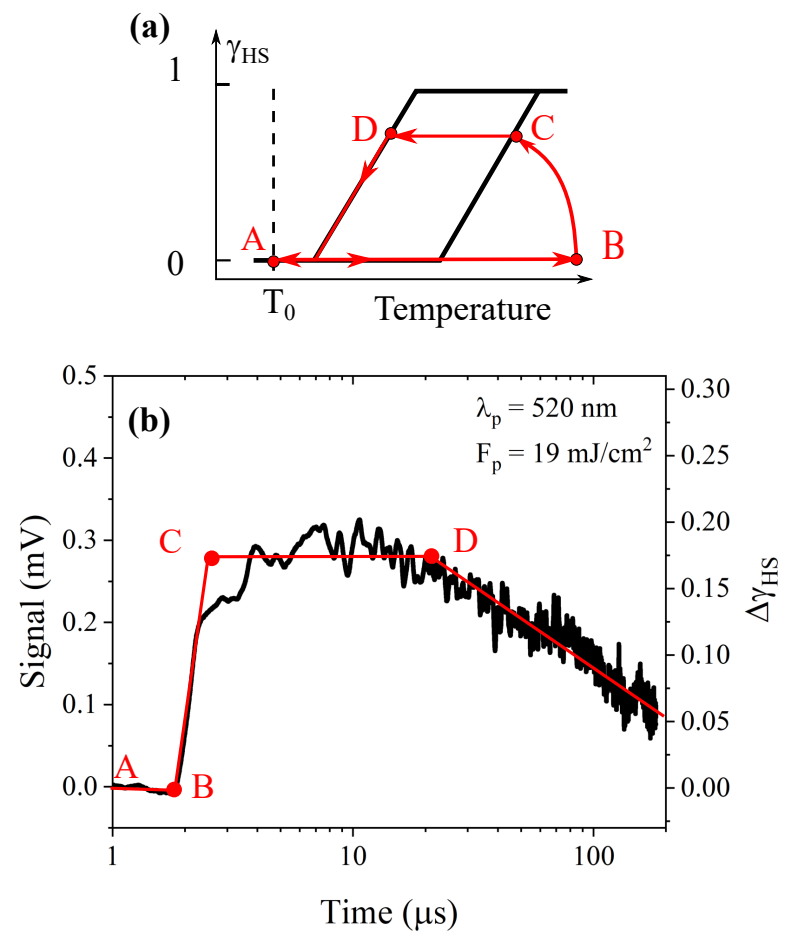

Figure 10: (a) Evolution of HS fraction in the $\left(\gamma_{H S}, T\right)$ hysteresis loop upon nanosecond pulsed excitation. (b) Evolution of the transmission signal during the evolution of $\gamma_{H S}$ in the $\left(\gamma_{H S}, T\right)$ hysteresis loop upon nanosecond pulsed excitation. The logarithm plot of the transmission signal is extracted from Fig. 8a. 
the absorption coefficient $\alpha\left(\lambda_{p}\right)$ versus the wavelength $\lambda_{p}$ and the characteristic size $L_{c}$ of the NPs. The second ones are the extrinsic parameters. These can be easily changed before or during an experiment. They are the thickness of the sample $e$, the laser beam diameter $\phi$, the peak power $I_{p}$ and the duration of the laser pulse $t_{p}$. All the characteristic lengths are associated with characteristic heat diffusion times. We can define the times $\tau_{d}^{N P}=\frac{L_{c}^{2}}{4 \pi^{2} D_{t h}}$, $\tau_{e}=\frac{e^{2}}{4 \pi^{2} D_{t h}}$ and $\tau_{\phi}=\frac{\phi^{2}}{2.4^{2} D_{t h}}$ it takes for heat to diffuse within a single NP, along the thickness and diameter of the sample, respectively.

The photo-thermal-switching kinetics depends on the speed at which temperature increases within the sample and therefore on the way heat is brought in the sample. Upon pulsed excitation of the sample, the heat can be considered to be brought almost instantaneously if the pulse duration $t_{p} \leq \tau_{d}^{N P}$. Under such a condition, typically achieved upon nanoseconds pulse excitation, the rise in temperature $\Delta T_{n s}$ can be readily computed knowing the laser fluence $E_{l} \sim \frac{t_{p} I_{p}}{\phi^{2}}$. It writes $\Delta T_{n s} \sim \frac{\alpha\left(\lambda_{p}\right) t_{p} I_{p}}{V \rho C_{p}}$ where $V$ is the volume of the sample shinned by the laser beam. If $\Delta T_{n s}$ is large enough, complete or partial photoswitching may be recorded on a time scale shorter than $\tau_{d}^{N P}$. Under this condition, the kinetics may be considered as the results of an ensemble NPs in which the growth of HS fraction $\gamma_{H S}$ occurs independently in each NP. A thermal photoswitching can also be recorded on time scale larger than $\tau_{d}^{N P}$, but then the growing of the HS state in a given NP is affected by the heat generated in a nearby NP due to heat diffusion. The subsequent evolution of the HS fraction $\Delta \gamma_{H S}$ depends on the evolution of the temperature and the position of the sample in the $\left(T, \gamma_{H S}\right)$ phase diagram. When the sample is initially set in the LS phase and below the thermal hysteresis loop, then if upon excitation $\Delta T_{n s}$ brings the sample above or along the ascending branch of the thermal hysteresis loop, $\Delta \gamma_{H S}$ growths, saturates and starts decreasing. The relaxation of $\Delta \gamma_{H S}$ starts when the heat generated within the sample can be dissipated out by the edges of the sample. For a thin sample and upon excitation, this happens after a lapse of time $\tau_{\text {relax }} \sim \tau_{e}$

Under QCW laser excitation, the pulse duration $t_{p} \gg \tau_{d}^{N P}$ and the increase in tem- 
perature $\Delta T_{Q C W}$ must be computed solving the heat equation. The solution of the latter strongly depends on the boundary conditions. Considering the sample is a thin disk that surfaces are kept at room temperature, we have shown that the maximal rise in temperature $\Delta T_{Q C W}$ resulting for absorption the laser power $I_{p}$ writes $\Delta T_{Q C W} \sim \frac{e^{2}}{D_{t h}} \frac{\alpha\left(\lambda_{p}\right) I_{p}}{V \rho C_{p}}$. At variance with the increase of temperature upon instantaneous heating, $\Delta T_{Q C W}$ is not linked to the pulse duration $t_{p}$ but depends on the thickness and the thermal diffusivity of the sample. The fact that the rise in temperature scales like $\Delta T_{Q C W} \sim e^{2}$ may account for the large increase in $\Delta \gamma_{H S}$ we reported previously shinning a $1 \mathrm{~mm}$ thick sample with laser power density of $15 \mathrm{~mW} \mathrm{~cm}{ }^{-2} \cdot{ }^{37}$ Indeed considering that the experimental conditions are very similar and that only the thickness of the sample is increased from $4 \mu \mathrm{m}$ to $1 \mathrm{~mm}$, then $\Delta T_{Q C W}(1 \mathrm{~mm}) \sim 10^{5} \Delta T_{Q C W}(4 \mu \mathrm{m})$ ! We have also evaluated the typical time $\tau_{Q C W} \sim \frac{\phi^{2}}{D_{t h}}$ it takes to achieve the $\Delta T_{Q C W}$. The expression of $\tau_{Q C W}$ emphasizes the size of the laser beam directly impacting the LS to HS photoswitching kinetics. For beam diameter of about $1 \mathrm{~mm}$ and conventional heat diffusivity of SCO compound the constant time for the temperature rise is $\tau_{Q C W} \sim 300 \mathrm{~ms}$. The latter is usually very large compared to the time it takes for the HS to grow within a NP. Hence, we can consider the growing of the HS state is due to an ensemble of NP coupled to each other by diffusion of heat within the sample. It is noteworthy to mention that as soon as the pump beam is stopped the temperature readily relaxes with the constant time $\tau_{\text {relax }} \sim \tau_{e}$. If the sample was initially within the thermal hysteresis loop $\Delta \gamma_{H S}$ achieved during photoexcitation remains otherwise $\Delta \gamma_{H S}$ relaxes back towards zero.

Finally, we compare the ratio between the temperature increase achieved upon nanoseconds or QCW pulse excitation with laser pulses having the same peak power. The latter ratio writes $\frac{\Delta T_{n s}}{\Delta T_{Q C W}} \sim \frac{t_{p} D_{t h}}{e^{2}} \sim \frac{t_{p}}{\tau_{e}}$ where $t_{p}$ is the duration of the nanosecond pulse. Since in the experiment we carried out $t_{p} \sim 10^{-8} s$ and $\tau_{e} \sim 10^{-4} s$ then $\frac{\Delta T_{n s}}{\Delta T_{Q C W}} \sim 10^{-4}$. This indicates that recording fast photoswitching requires nanosecond laser pulses with high peak power, the latter being limited by the optical damage threshold of the material. Table. 1 
summarizes all the key parameters affecting the kinetics and rise in temperature.

Table 1: The dependence of photo-switching time, temperature increase and relaxation time on the relation between the pulse duration $t_{p}$ and the heat diffusion characteristic time in a single SCO NP $\tau_{d}^{N P}$.

Photo-switching Relaxation outside the thermal hysteresis loop

\begin{tabular}{lcc}
\hline$t_{p} \leq \tau_{d}^{N P}$ & Instantaneous heating & Occurs after $t \geq \tau_{e}=\frac{e^{2}}{4 \pi^{2} D_{t h}}$ \\
& Rise time $\sim t_{p} \sim 10^{-8} s$ & $\tau_{e} \sim 10^{-4} s$ \\
& Temperature $\Delta T_{n s} \sim \frac{\alpha\left(\lambda_{p}\right) t_{p} I_{p}}{V \rho C_{p}}$ & \\
\hline$t_{p} \gg \tau_{d}^{N P}$ & Non-instantaneous heating & Instantaneous relaxation \\
& Rise time $\tau_{\phi}=\frac{\phi^{2}}{2.4^{2} D_{t h}}$ & \\
Temperature $\Delta T_{Q C W} \sim \frac{e^{2}}{D_{t h}} \frac{\alpha\left(\lambda_{p}\right) I_{p}}{V \rho C_{p}}$ &
\end{tabular}

\section{Conclusions}

We have investigated the photoswitching process between the LS and HS states taking place in a thin layer of SCO of polymeric triazole based iron(II) complexes of [Fe(Htrz $\left.)_{2} \operatorname{trz}\right]\left(\mathrm{BF}_{4}\right)$ and nanohybrid made of AuNRs coated in a shell of this SCO polymeric $\left[\mathrm{Fe}(\mathrm{Htrz})_{2}\right.$ trz $]\left(\mathrm{BF}_{4}\right)$. The experiments were performed setting the sample in the LS state and exciting the samples with near-infrared quasi-continuous wave or nanosecond pulses, tunable in visible to nearinfrared range. The photoswitching experiments were performed recording the evolution of transmission of the sample near the SPR of the AuNRs. When a QCW pulse with peak power of few hundreds of milliwatts excites the sample close to the SPR of the AuNRs, the photoswitching happens on few hundreds of millisecond time. However, the latter process is only recorded when the temperature of the samples is set near the ascending branch of the hysteresis loop. Hence, upon excitation the sample is brought in a HS-LS mixture state within the thermal hysteresis loop. We also run similar experiments setting the temperature of the samples below the thermal hysteresis and exciting them with a nanosecond pulse. Under these conditions, we recorded both the photoswitching from the LS to HS state as well as the relaxation from the HS to LS state. The photoswitching amplitude and time 
constant depend on the laser fluence and absorption of the samples at the exciting pulse wavelength. A photoswitching time constant down to 20 ns has been recorded, indicating that, as expected one can very rapidly switch a non-negligible amount of the studied samples from the LS to HS state. For the nanohybrid, it was shown that the nanosecond pulse energy required to switch the sample from the LS to HS state decreases as one tune the central frequency of the nanosecond laser pulse toward the SPR of the AuNRs. We have also proposed a simple model that accounts well for this ensemble of results. It makes it possible to evaluate the kinetics and rise in temperature within the sample upon QCW or nanosecond pulsed excitation. The amplitude of the temperature rises nicely accounts for the change upon LS to HS photoswitching. The evolution and rise in temperature within the sample were also used to propose a scenario that accounts for the reported experiments. This simple model also shines some light on some parameters of the sample such as the thicknesses and absorption that impact the rise and relaxation in temperature and therefore on the photoswitching kinetics. It happens within tens of nanoseconds for nanosecond pulses. We have also shown that the experiment using nanosecond pulse make it possible to analyze the photo-switching within individual NPs whereas the ones with quasi-continuous wave excitation reveal the photo-switching within an ensemble of NPs.

\section{Supporting Information Available}

Solution of the heat equation of a thin disk photo excited by a quasi continuous wave.

\section{Acknowledgement}

CNRS, University of Bordeaux, Nouvelle Aquitaine Region and the ANR program (HEROES ANR-17-CE09-0010-01) are acknowledged for the fundings. 


\section{References}

(1) Cambi, L.; Szegö, L. Über die magnetische Susceptibilität der komplexen Verbindungen. Berichte der deutschen chemischen Gesellschaft (A and B Series) 1931, 64, 2591-2598.

(2) Stoufer, R. C.; Busch, D. H.; Hadley, W. B. UNUSUAL MAGNETIC PROPERTIES OF SOME SIX-COÖRDINATE COBALT(II) COMPLEXES1 —ELECTRONIC ISOMERS. Journal of the American Chemical Society 1961, 83, 3732-3734.

(3) König, E.; Madeja, K. 5T2-1A1 Equilibriums in some iron(II)-bis(1,10-phenanthroline) complexes. Inorganic Chemistry 1967, 6, 48-55.

(4) Gütlich, P.; Goodwin, H. Spin Crossover in Transition Metal Compounds I; Topics in Current Chemistry; Springer Berlin Heidelberg, 2004.

(5) Bousseksou, A.; Molnár, G.; Salmon, L.; Nicolazzi, W. Molecular spin crossover phenomenon: recent achievements and prospects. Chem. Soc. Rev. 2011, 40, 3313-3335, Publisher: The Royal Society of Chemistry.

(6) Murray, K. S.; Oshio, H.; Real, J. A. Spin-crossover complexes. European Journal of Inorganic Chemistry 2013, 577-580.

(7) Gütlich, P.; Gaspar, A. B.; Garcia, Y. Spin state switching in iron coordination compounds. Beilstein journal of organic chemistry 2013, 9, 342-391.

(8) Halcrow, M. A. Spin-crossover materials: properties and applications; John Wiley \& Sons, 2013.

(9) Hayami, S.; Holmes, S. M.; Halcrow, M. A. Spin-state switches in molecular materials chemistry. Journal of Materials Chemistry C 2015, 3, 7775-7778.

(10) Dugay, J.; Giménez-Marqués, M.; Kozlova, T.; Zandbergen, H. W.; Coronado, E.; van der Zant, H. S. J. Spin Switching in Electronic Devices Based on 2D Assemblies of Spin-Crossover Nanoparticles. Advanced Materials 2015, 27, 1288-1293. 
(11) Dugay, J.; Giménez-Marqués, M.; Venstra, W. J.; Torres-Cavanillas, R.; Sheombarsing, U. N.; Manca, N.; Coronado, E.; van der Zant, H. S. J. Sensing of the Molecular Spin in Spin-Crossover Nanoparticles with Micromechanical Resonators. The Journal of Physical Chemistry C 2019, 123, 6778-6786.

(12) Coronado, E.; Giménez-Marqués, M.; Espallargas, G. M.; Brammer, L. Tuning the magneto-structural properties of non-porous coordination polymers by $\mathrm{HCl}$ chemisorption. Nature communications 2012, 3, 828 .

(13) Cavallini, M. Status and perspectives in thin films and patterning of spin crossover compounds. Phys. Chem. Chem. Phys. 2012, 14, 11867-11876.

(14) Salmon, L.; Molnár, G.; Zitouni, D.; Quintero, C.; Bergaud, C.; Micheau, J.-C.; Bousseksou, A. A novel approach for fluorescent thermometry and thermal imaging purposes using spin crossover nanoparticles. Journal of Materials Chemistry 2010, 20, $5499-5503$.

(15) Kahn, O.; Kröber, J.; Jay, C. Spin transition molecular materials for displays and data recording. Advanced Materials 1992, 4, 718-728.

(16) Kroeber, J.; Audiere, J.-P.; Claude, R.; Codjovi, E.; Kahn, O.; Haasnoot, J. G.; Groliere, F.; Jay, C.; Bousseksou, A. Spin transitions and thermal hysteresis in the molecular-based materials [Fe (Htrz) 2 (trz)](BF4) and [Fe (Htrz) 3](BF4) 2. cntdot. $\mathrm{H} 2 \mathrm{O}$ (Htrz= 1, 2, 4-4H-triazole; trz= 1, 2, 4-triazolato). Chemistry of materials 1994, 6, 1404-1412.

(17) Roubeau, O. Triazole-Based One-Dimensional Spin-Crossover Coordination Polymers. Chemistry - A European Journal 2012, 18, 15230-15244.

(18) Herrera, J. M.; Titos-Padilla, S.; Pope, S. J. A.; Berlanga, I.; Zamora, F.; Delgado, J. J.; Kamenev, K. V.; Wang, X.; Prescimone, A.; Brechin, E. K.; Colacio, E. Studies on 
bifunctional Fe(II)-triazole spin crossover nanoparticles: time-dependent luminescence, surface grafting and the effect of a silica shell and hydrostatic pressure on the magnetic properties. Journal of Materials Chemistry C 2015, 3, 7819-7829, Publisher: The Royal Society of Chemistry.

(19) Coronado, E.; Galán-Mascarós, J. R.; Monrabal-Capilla, M.; García-Martínez, J.; Pardo-Ibáñez, P. Bistable spin-crossover nanoparticles showing magnetic thermal hysteresis near room temperature. Advanced Materials 2007, 19, 1359-1361.

(20) Titos-Padilla, S.; Herrera, J. M.; Chen, X.-W.; Delgado, J. J.; Colacio, E. Bifunctional Hybrid SiO2 Nanoparticles Showing Synergy between Core Spin Crossover and Shell Luminescence Properties. Angewandte Chemie International Edition 2011, 50, 32903293, _eprint: https://onlinelibrary.wiley.com/doi/pdf/10.1002/anie.201007847.

(21) Herrera, J. M.; Titos-Padilla, S.; Pope, S. J.; Berlanga, I.; Zamora, F.; Delgado, J. J.; Kamenev, K. V.; Wang, X.; Prescimone, A.; Brechin, E. K., et al. Studies on bifunctional Fe (II)-triazole spin crossover nanoparticles: time-dependent luminescence, surface grafting and the effect of a silica shell and hydrostatic pressure on the magnetic properties. Journal of Materials Chemistry C 2015, 3, 7819-7829.

(22) Torres-Cavanillas, R.; Lima-Moya, L.; Tichelaar, F. D.; Zandbergen, H. W.; GiménezMarqués, M.; Coronado, E. Downsizing of robust Fe-triazole@SiO2 spin-crossover nanoparticles with ultrathin shells. Dalton Transactions 2019, 48, 15465-15469, Publisher: The Royal Society of Chemistry.

(23) Li, Z.-H.; Wang, Y.-X.; Han, W.-K.; Zhu, W.; Li, T.; Li, Z.; Ren, X.; Gu, Z.-G. Integrating spin-crossover nanoparticles with silver nanowires: toward magnetic and conductive bifunctional nanomaterials. New Journal of Chemistry 2017, 41, 1006210068, Publisher: The Royal Society of Chemistry. 
(24) Salmon, L.; Catala, L. Spin-crossover nanoparticles and nanocomposite materials. Comptes Rendus Chimie 2018, 21, 1230-1269.

(25) Hauser, A. Reversibility of light-induced excited spin state trapping in the Fe(ptz)6(BF4)2, and the Zn1-xFex(ptz)6(BF4)2 spin-crossover systems. Chemical Physics Letters 1986, 124, 543-548.

(26) Parpiiev, T.; Servol, M.; Lorenc, M.; Chaban, I.; Lefort, R.; Collet, E.; Cailleau, H.; Ruello, P.; Daro, N.; Chastanet, G., et al. Ultrafast non-thermal laser excitation of gigahertz longitudinal and shear acoustic waves in spin-crossover molecular crystals [Fe (PM-AzA) 2 (NCS) 2]. Applied Physics Letters 2017, 111, 151901.

(27) Létard, J.-F.; Guionneau, P.; Rabardel, L.; Howard, J. A.; Goeta, A. E.; Chasseau, D.; Kahn, O. Structural, magnetic, and photomagnetic studies of a mononuclear iron (II) derivative exhibiting an exceptionally abrupt spin transition. Light-induced thermal hysteresis phenomenon. Inorganic chemistry 1998, 37, 4432-4441.

(28) Marino, A.; Servol, M.; Bertoni, R.; Lorenc, M.; Mauriac, C.; Létard, J.-F.; Collet, E. Femtosecond optical pump-probe reflectivity studies of spin-state photo-switching in the spin-crossover molecular crystals [Fe(PM-AzA)2(NCS)2]. Polyhedron 2013, 66, 123-128, ICMM 2012.

(29) Fouché, O.; Degert, J.; Jonusauskas, G.; Balde, C.; Desplanches, C.; Létard, J.-F.; Freysz, E. Laser induced spin state transition: Spectral and temporal evolution. Chemical Physics Letters 2009, 469, 274-278.

(30) Fouché, O.; Degert, J.; Jonusauskas, G.; Daro, N.; Létard, J.-F.; Freysz, E. Mechanism for optical switching of the spin crossover [Fe (NH 2-trz) 3](Br) 2. 3H $2 \mathrm{O}$ compound at room temperature. Physical Chemistry Chemical Physics 2010, 12, 3044-3052.

(31) Gallé, G.; Deldicque, D.; Degert, J.; Forestier, T.; Létard, J.-F.; Freysz, E. Room 
temperature study of the optical switching of a spin crossover compound inside its thermal hysteresis loop. Applied Physics Letters 2010, 96, 041907.

(32) Gallé, G.; Degert, J.; Mauriac, C.; Etrillard, C.; Létard, J.-F.; Freysz, E. Nanosecond study of spin state transition induced by a single nanosecond laser shot on [Fe(NH2trz)3] compounds inside and outside their thermal hysteresis loops. Chemical Physics Letters 2010, 500, 18-22.

(33) Gallé, G.; Etrillard, C.; Degert, J.; Guillaume, F.; Létard, J.-F.; Freysz, E. Study of the fast photoswitching of spin crossover nanoparticles outside and inside their thermal hysteresis loop. Applied Physics Letters 2013, 102, 063302.

(34) Moulet, L.; Daro, N.; Mornet, S.; Vilar-Vidal, N.; Chastanet, G.; Guionneau, P. Grafting of gold onto spin-crossover nanoparticles: SCO@Au. Chemical Communications 2016, 52, 13213-13216.

(35) Qiu, D.; Gu, L.; Sun, X.-L.; Ren, D.-H.; Gu, Z.-G.; Li, Z. SCO@ SiO 2@ Au core-shell nanomaterials: enhanced photo-thermal plasmonic effect and spin-crossover properties. RSC advances 2014, 4, 61313-61319.

(36) Suleimanov, I.; Sánchez Costa, J.; Molnár, G.; Salmon, L.; Bousseksou, A. The photothermal plasmonic effect in spin crossover@silica-gold nanocomposites. Chemical Communications 2014, 50, 13015-13018.

(37) Palluel, M.; Tran, N. M.; Daro, N.; Buffière, S.; Mornet, S.; Freysz, E.; Chastanet, G. The Interplay between Surface Plasmon Resonance and Switching Properties in Gold@Spin Crossover Nanocomposites. Advanced Functional Materials 2020, 30, 2000447.

(38) Torres-Cavanillas, R.; Sanchis-Gual, R.; Dugay, J.; Coronado-Puchau, M.; GiménezMarqués, M.; Coronado, E. Design of Bistable Gold@Spin-Crossover Core-Shell 
Nanoparticles Showing Large Electrical Responses for the Spin Switching. Advanced Materials 2019, 31, 1900039.

(39) Roubeau, O.; Castro, M.; Burriel, R.; Haasnoot, J. G.; Reedijk, J. Calorimetric investigation of triazole-bridged Fe (II) spin-crossover one-dimensional materials: measuring the cooperativity. The Journal of Physical Chemistry B 2011, 115, 3003-3012.

(40) Zhang, W. Experimental and computational analysis of random cylinder packings with applications; LSU Doctoral Dissertations. 163, 1993. 